\title{
Influência do sulfato de bário nas características de cerâmica vermelha incorporada com resíduo oleoso inertizado
}

\section{(Influence of barium sulfate on the characteristics of red ceramic incorporated with oily waste)}

\author{
F. A. N. Silva ${ }^{1}$ S. N. Monteiro', C. M. F. Vieira ${ }^{2}$, M. F. S. Lopes ${ }^{3}$ \\ ${ }^{1}$ Centro Federal de Educação Tecnológica - CEFET CAMPOS \\ ${ }^{2}$ CCT - LAMAV, UENF, Av. Alberto Lamego, 2000 \\ Campos dos Goytacazes, RJ 28013-602 \\ ${ }^{3}$ Pontifícia Universidade Católica do Rio de Janeiro, PUC-RJ, Rio de Janeiro, RJ \\ vieira@uenf.br
}

Resumo

\begin{abstract}
A incorporação em cerâmicas de resíduos como borras resultantes da extração, transporte ou refino de petróleo evita seu lançamento no meio ambiente. Por outro lado, é importante avaliar as alterações tecnológicas causadas pela adição do resíduo, sobretudo, no caso de comprometer a qualidade do produto cerâmico. Neste trabalho estudou-se a microestrutura de um material cerâmico contendo 0, 10, 15 e 20\% em peso de um resíduo denominado borra de petróleo encapsulada, queimado em temperaturas que variam de 850 a $1100{ }^{\circ} \mathrm{C}$. A microanálise do material foi realizada através de espectrometria por dispersão de energia (EDS) de modo a complementar a análise topográfica feita por microscopia eletrônica de varredura (MEV), para possibilitar a identificação dos elementos químicos presentes, bem como partículas de segunda fase. Os resultados demonstraram que a adição desta borra de petróleo encapsulada provoca alterações tanto na composição química quanto na microestrutura do material cerâmico. Partículas de formato e características distintas das observadas na cerâmica sem adição de resíduo, principalmente partículas de sulfato de bário, foram mapeadas e sua influência discutida.
\end{abstract}

Palavras-chave: resíduo oleoso, sulfato de bário, cerâmica incorporada.

\begin{abstract}
The incorporation into ceramics of residues, such as oily wastes from extraction, transport and refining of petroleum is a way to avoid their disposal to the environment. On the other hand, it is important to evaluate the technological changes caused by the addition of the residue, mainly, in the case that it could compromise the quality of the ceramic product. In this work, the microstructure resulting from the incorporation with 0, 10, 15 and 20 wt.\% of a residue, known as encapsulated petroleum waste, into a ceramic material that was fired at temperatures in the range of 850 to $1100^{\circ} \mathrm{C}$, was investigated. The microanalysis of the material was performed by Energy Dispersed Spectroscopy (EDS) to complement the topographic carried out by scanning electron microscopy (SEM). This was done to make it possible the identification of chemical elements as well as second phase particles. The results showed that the incorporation of this encapsulated petroleum waste induced changes in both, the chemical composition and the microstructure of the ceramic material.
\end{abstract}

Keywords: oily waste, barium sulfate, incorporated ceramic.

\section{INTRODUÇÃO}

A sociedade moderna valoriza processos que envolvam reciclagem de materiais. Deste modo, os investimentos relacionados ao aproveitamento de resíduos tornaram-se essenciais ao desenvolvimento como forma, inclusive, de proteção ambiental. Por exemplo, a indústria petrolífera gera resíduos com características e composições bastante diversificadas que podem ser incorporados a outros materiais como alternativa ao simples lançamento no meio ambiente. O resíduo dos separadores de água e óleo das plataformas marítimas, após ser submetido ao processo de inertização, é conhecido como borra de petróleo encapsulada. Este resíduo vem sendo estudado visando um possível aproveitamento como matéria prima na confecção de cerâmica vermelha [1-7]. Entretanto ainda perduram dúvidas quanto ao efeito da adição destes resíduos na estrutura do produto acabado. 
O município de Campos dos Goytacazes, que hoje se encontra como o principal centro produtor de blocos de vedação do Estado do Rio de Janeiro, apresenta este setor em fase de evolução tecnológica visando produtos de maior valor agregado tais como de telhas, pisos rústicos e blocos estruturais. A borra de petróleo encapsulada, classificada pelos órgãos ambientais como classe II (não inerte) vem sendo avaliada como um possível aditivo para aprimorar não só os produtos mas também o processamento da indústria cerâmica regional [4-6].

Pesquisas demonstram que a utilização de resíduos oleosos na fabricação de cerâmicas vermelhas, em geral, acarreta melhoria em suas propriedades $[1,2,7]$. Estas pesquisas forneceram dados científicos e tecnológicos que podem auxiliar na avaliação da qualidade dos produtos cerâmicos obtidos após incorporação de borra de petróleo inertizada à massa cerâmica convencionalmente utilizada pela indústria.

Artigos recentes abordaram as vantagens e dificuldades da incorporação do resíduo dos separadores de água e óleo das plataformas marítimas, tanto inertizado [6] quanto cru [7]. Entretanto, ainda persistem alguns pontos controversos. Souza e Holanda [6] incorporaram até 20\% de resíduo oleoso inertizado e indicaram que a redução observada na resistência mecânica estaria associada à partículas de $\mathrm{BaSO}_{4}$ (barita) e $\mathrm{SiO}_{2}$ (quartzo). Isto foi atribuído ao efeito deletério de trincas e porosidades introduzidas na microestrutura devido às fracas ligações destas partículas não-plásticas com a matriz argilosa [6]. A dilatação diferencial e a quebra destas partículas poderiam também contribuir para a formação de vazios microestruturais e assim comprometeriam a resistência mecânica.

Por outro lado, Monteiro e Vieira [7] mostraram que a adição do resíduo oleoso cru, isto é, sem tratamento de inertização, acarreta um aumento na resistência mecânica para até $10 \%$ de resíduo incorporado. A razão para esta melhoria mecânica foi atribuída a um melhor empacotamento das partículas de argila na massa processada antes da queima, devido à ação lubrificante de filmes de óleo [2]. Para maiores adições de resíduo, o conteúdo oleoso do resíduo, além do filme, formaria bolsas de hidrocarbonetos que, após a queima, resultariam em poros. $\mathrm{O}$ balanço final seria então desfavorável à resistência mecânica para teores acima de $10 \%$ de resíduo oleoso. Assim, as bolsas de óleo que na queima viram vazios poderiam ser mais prejudiciais à resistência do que as partículas de $\mathrm{BaSO}_{4}$ e $\mathrm{SiO}_{2}$.

Tendo em vista estes pontos controversos, o objetivo do presente trabalho foi realizar uma ampla investigação da microestrutura de cerâmicas argilosas incorporadas com um resíduo oleoso inertizado. Esta investigação foi conduzida através de observações detalhadas da superfície de fratura com técnica de microscopia eletrônica de varredura associada à microanálise realizada através de mapeamento elementar ou por identificação dos picos de difração de raios X.

\section{MATERIAIS E MÉTODOS}

O resíduo oleoso utilizado no presente trabalho foi cedido pela Petrobrás. Este resíduo é identificado como inertizado, tendo sido aprovado nos testes ambientais para uso como material para cobertura de aterro sanitário [8]. A origem deste resíduo é uma borra pastosa obtida no processo de separação de água e óleo realizado na plataforma marítima após a extração do petróleo. Em seguida ao desembarque na cidade de Macaé, esta borra sofre homogeneização e tratamento de inertização. A inertização consiste na adição de $20 \%$ em peso de bentonita que serve como agente encapsulante do conteúdo oleoso. O material aguarda oito dias para sofrer inertização por encapsulamento ocorrendo então clareamento na cor e alteração da consistência pastosa para uma textura arenosa. A análise química do resíduo oleoso inertizado está apresentada na Tabela I. Observa-se que o resíduo é composto predominantemente por $\mathrm{SiO}_{2}$, na forma de quartzo, e $\mathrm{SO}_{3}$ e $\mathrm{BaO}$, possivelmente associados ao composto $\mathrm{BaSO}_{4}$ forma de barita.

A quantidade estimada de hidrocarbonetos na borra é de 9,3\% em peso e a quantidade de água é de 4,3\% em peso.

Vale aqui mencionar que uma análise química indicada pela Petrobrás [8] não relata a existência de alguns dos metais cujos óxidos se encontram na Tabela I. Provavelmente a análise da Petrobrás visou somente elementos e compostos químicos de maior toxidade. Entretanto, os metais pesados bário e estrôncio não aparecem na análise da Petrobrás, embora tenham teores significativos nas medidas realizadas para o presente trabalho.

Foram preparados corpos de prova por prensagem uniaxial a $20 \mathrm{MPa}$ com incorporação de 0, 10, 15 e $20 \%$ em peso de resíduo em argila caulinítica [4]. Os corpos de prova foram inicialmente secos em estufa até peso constante. Em seguida, foram queimados em forno de laboratório em temperaturas variando de 850 a $1100{ }^{\circ} \mathrm{C}$, em intervalos de $50^{\circ} \mathrm{C}$.

A superfície de fratur a dos corpos de prova queimados foi observada por microscopia eletrônica de varredura, utilizando-se microscópio Zeiss, modelo DSM 960. A microanálise de material foi realizada por espectrometria por dispersão de energia (EDS), para complementar a análise topográfica e identificar os elementos químicos presentes ou partículas de segundas fases.

Tabela I - Composição química do resíduo oleoso inertizado (\% em peso).

[Table I-Chemical composition of the inert oily waste (wt.\%).]

\begin{tabular}{ccccccccccccccc}
\hline $\mathrm{SiO}_{2}$ & $\mathrm{SO}_{3}$ & $\mathrm{BaO}$ & $\mathrm{Fe}_{2} \mathrm{O}_{3}$ & $\mathrm{Al}_{2} \mathrm{O}_{3}$ & $\mathrm{CaO}$ & $\mathrm{K}_{2} \mathrm{O}$ & $\mathrm{NaO}$ & $\mathrm{SrO}$ & $\mathrm{MgO}$ & $\mathrm{TiO}_{2}$ & $\mathrm{CuO}$ & $\mathrm{ZnO}$ & $\mathrm{Cr}_{2} \mathrm{O}_{3}$ & $\mathrm{MnO}_{2}$ \\
\hline 40,0 & 11,5 & 10,1 & 7,3 & 6,6 & 5,6 & 1,5 & 1,1 & 1,0 & 0,7 & 0,4 & 0,2 & 0,2 & 0,1 & 0,1 \\
\hline
\end{tabular}




\section{RESULTADOS E DISCUSSÃO}

As Figs. 1 a 3 mostram a superfície de fratura da cerâmica sem incorporação de resíduo, destacando partículas, indicadas pela letra $\mathbf{P}$, submetidas à análise de EDS. Observa-se tipicamente nestas figuras uma estrutura porosa, contendo partículas ricas, principalmente, em ferro, silício e titânio. Estas partículas mostram-se bem aderida à massa argilosa e aparentemente foram rompidas durante a fratura do material. Com a elevação da temperatura, Fig. 3, as partículas apresentam-se mais efetivamente ligadas à matriz de fase vítrea.
Outras partículas ricas em silício, provavelmente grãos de quartzo, foram encontradas em todas as amostras do material sem adição de borra encapsulada de petróleo. A Fig. 4 apresenta uma destas partículas na cerâmica queimada a $1100{ }^{\circ} \mathrm{C}$, bem aderida à matriz, e que sofreu ruptura típica concoidal durante o processo de fratura do corpo cerâmico.

Mapeamento por EDS das superfícies de fratura revelou para todas as temperaturas de queima, uma distribuição uniforme de elementos com pequenas partículas (brancas), como ilustra a Fig. 5.

Após incorporação do resíduo de borra inertizada,
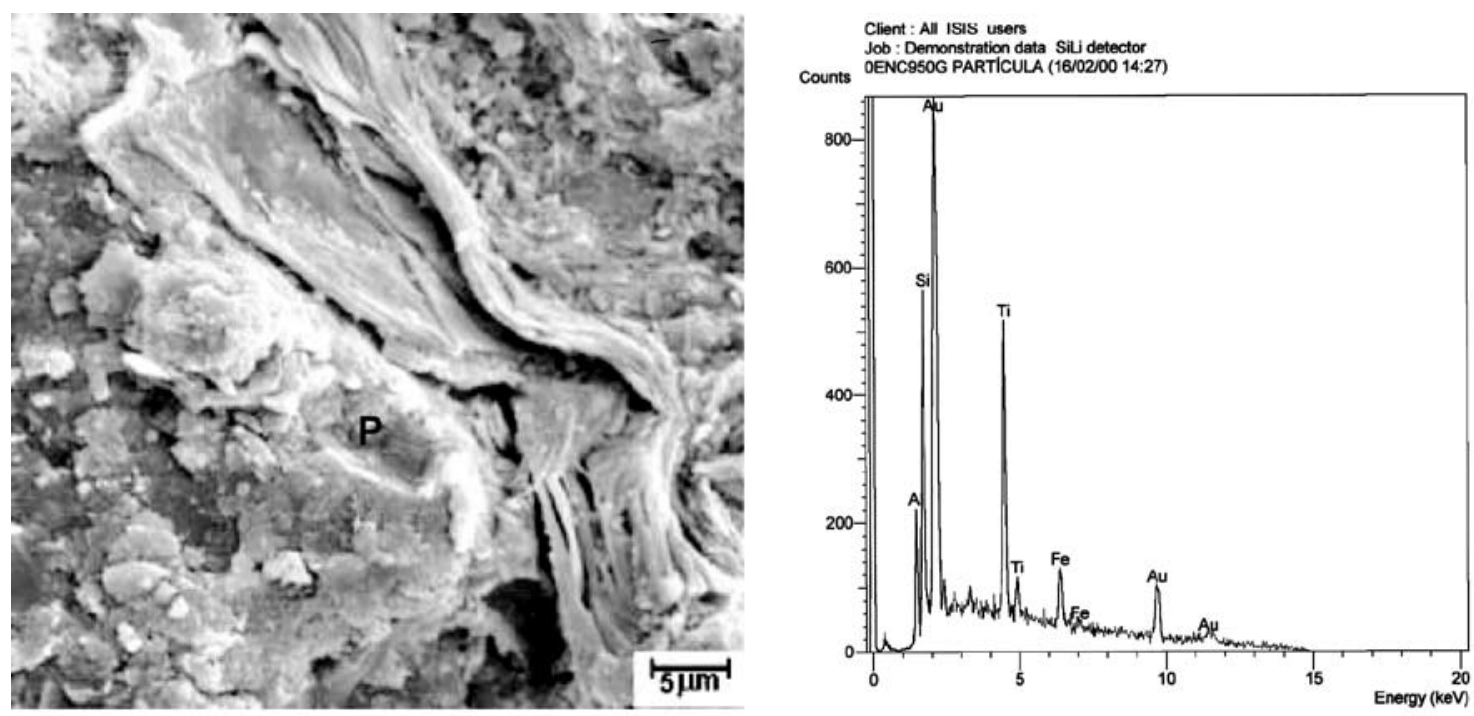

Figura 1: Micrografia obtida por MEV da cerâmica sem resíduo oleoso inertizado queimada a $950{ }^{\circ} \mathrm{C}$ com EDS mostrando partícula P composta predominantemente de Si e Ti.

[Figure 1: SEM micrograph of the waste-free clay fired at $950^{\circ} \mathrm{C}$ with EDS showing a particle $\boldsymbol{P}$ predominantly composed of Si and Ti.]
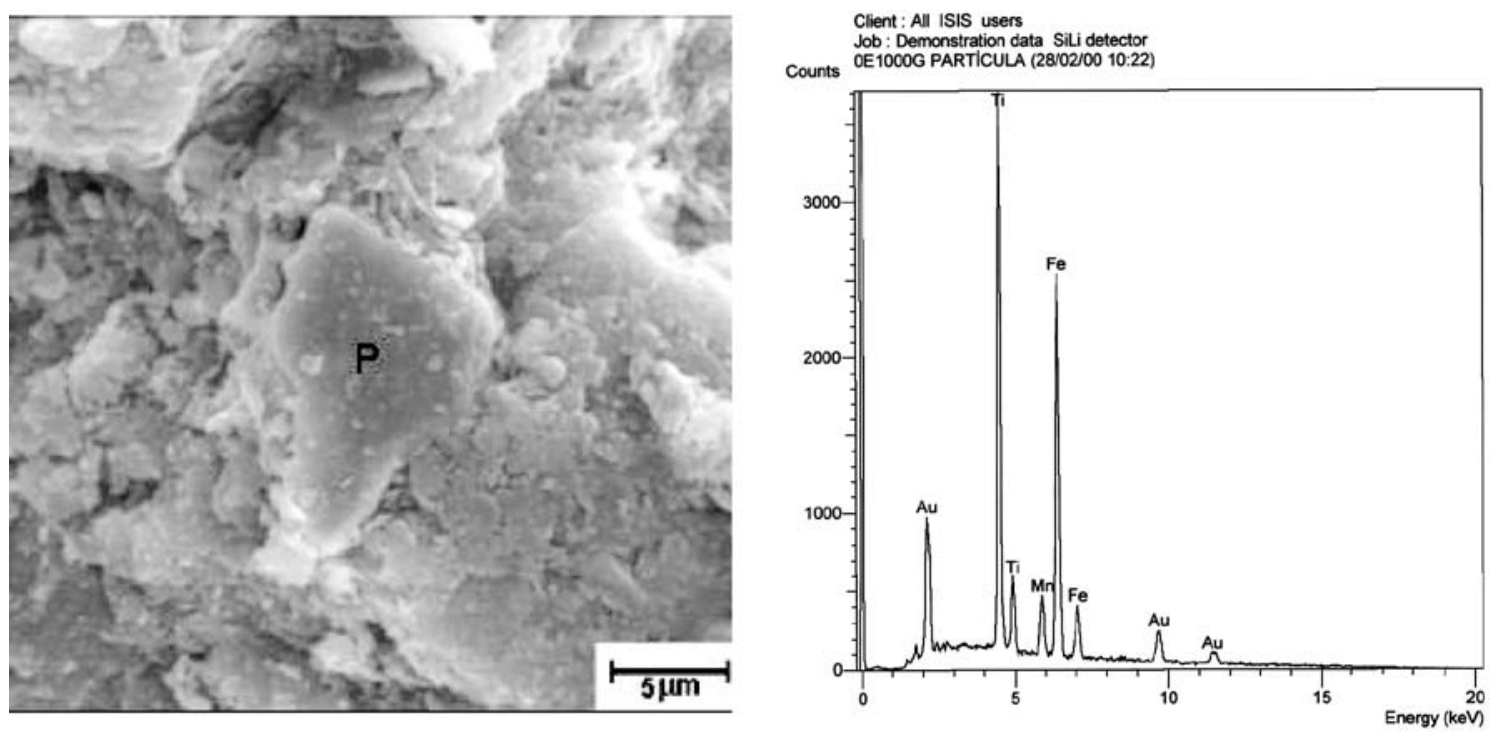

Figura 2: Micrografia obtida por MEV da cerâmica sem resíduo oleoso inertizado queimada a $1000{ }^{\circ} \mathrm{C}$ com EDS mostrando partícula $\mathrm{P}$ composta predominantemente de Ti e Fe.

[Figure 2: SEM micrograph of the waste-free clay fired at $1000^{\circ} \mathrm{C}$ with EDS showing a particle $\mathbf{P}$ predominantly composed of Ti and Fe.] 

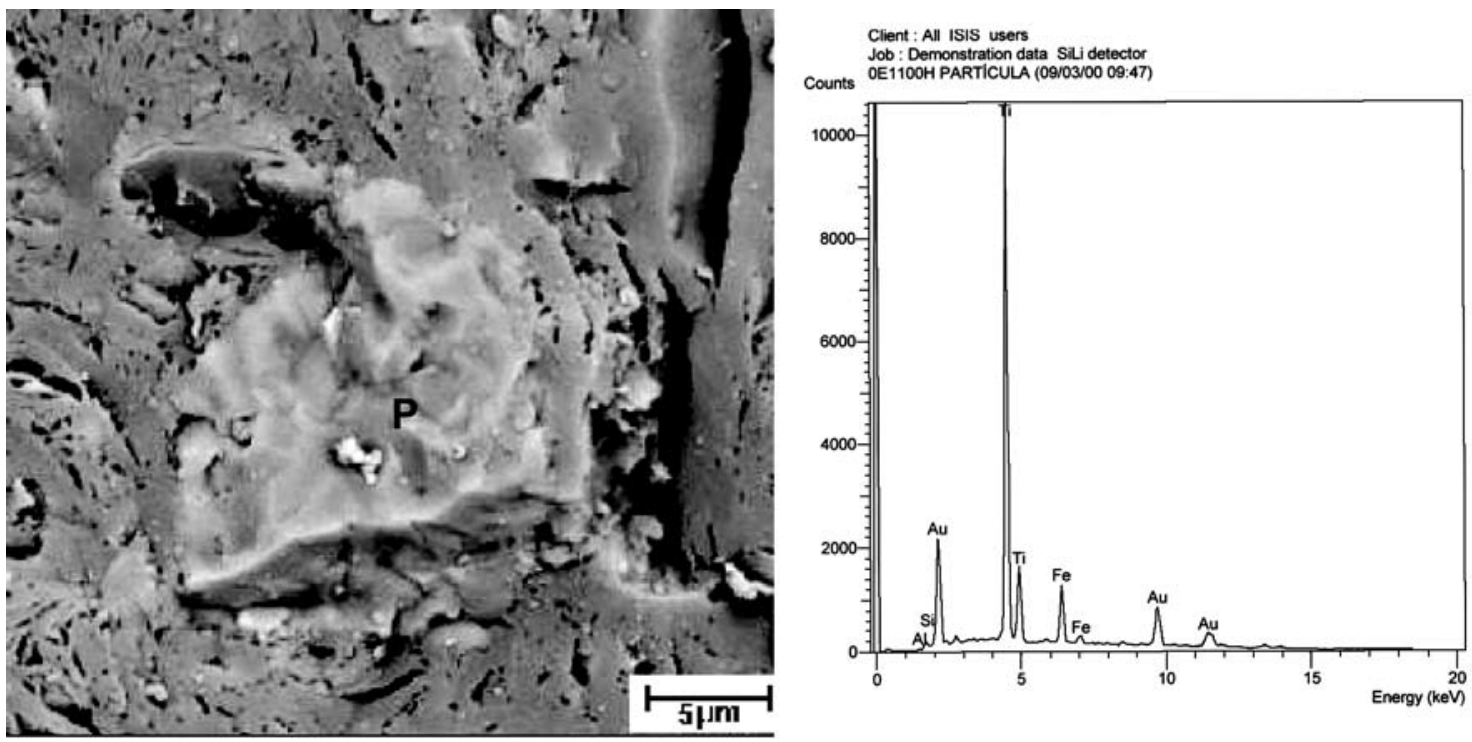

Figura 3: Micrografia obtida por MEV da cerâmica sem resíduo oleoso inertizado queimada a $1100{ }^{\circ} \mathrm{C}$ com EDS mostrando partícula $\mathrm{P}$ composta predominantemente de Ti.

[Figure 3: SEM micrograph of the waste-free clay fired at $1100^{\circ} \mathrm{C}$ with EDS showing a particle $\boldsymbol{P}$ predominantly composed of Ti.]

Figs. 6 a 8, foram observadas também partículas distintas das anteriormente descritas. Isto é, além das partículas já previstas, ricas principalmente em titânio, silício e ferro, constatou-se a existência de partículas de composição e aspectos bastante diferenciados.

A partícula da Fig. 6, de acordo com o seu espectro EDS, é rica em bário e enxofre. Esta partícula é provavelmente sulfato de bário (BaSO $)$ resultante da incorporação de 20\% de borra encapsulada e queimada a $1050{ }^{\circ} \mathrm{C}$. Na Fig. 7, correspondente a uma cerâmica incorporada com 15\% de borra e queimada a $1100{ }^{\circ} \mathrm{C}$, observam-se duas partículas aparentemente iguais. Entretanto, os respectivos espectros EDS indicam compostos distintos. Assim, a partícula P1 seria $\mathrm{BaSO}_{4}$ enquanto $\mathrm{P} 2$ seria quartzo $\left(\mathrm{SiO}_{2}\right)$.

A Fig. 8, correspondente à borra encapsulada pura (100\% de resíduo oleoso) queimada a $950^{\circ} \mathrm{C}$, apresenta diversas partículas identificadas como $\mathrm{BaSO}_{4}$. Além disto, um espectro geral da borra confirma o teor relativamente elevado, Tabela I, de $\mathrm{BaSO}_{4}$ juntamente com óxido de $\mathrm{Si}$ e Fe. Como as partículas de $\mathrm{BaSO}_{4}$ não foram observadas, Figs. 1 a 3, nas cerâmicas sem adição
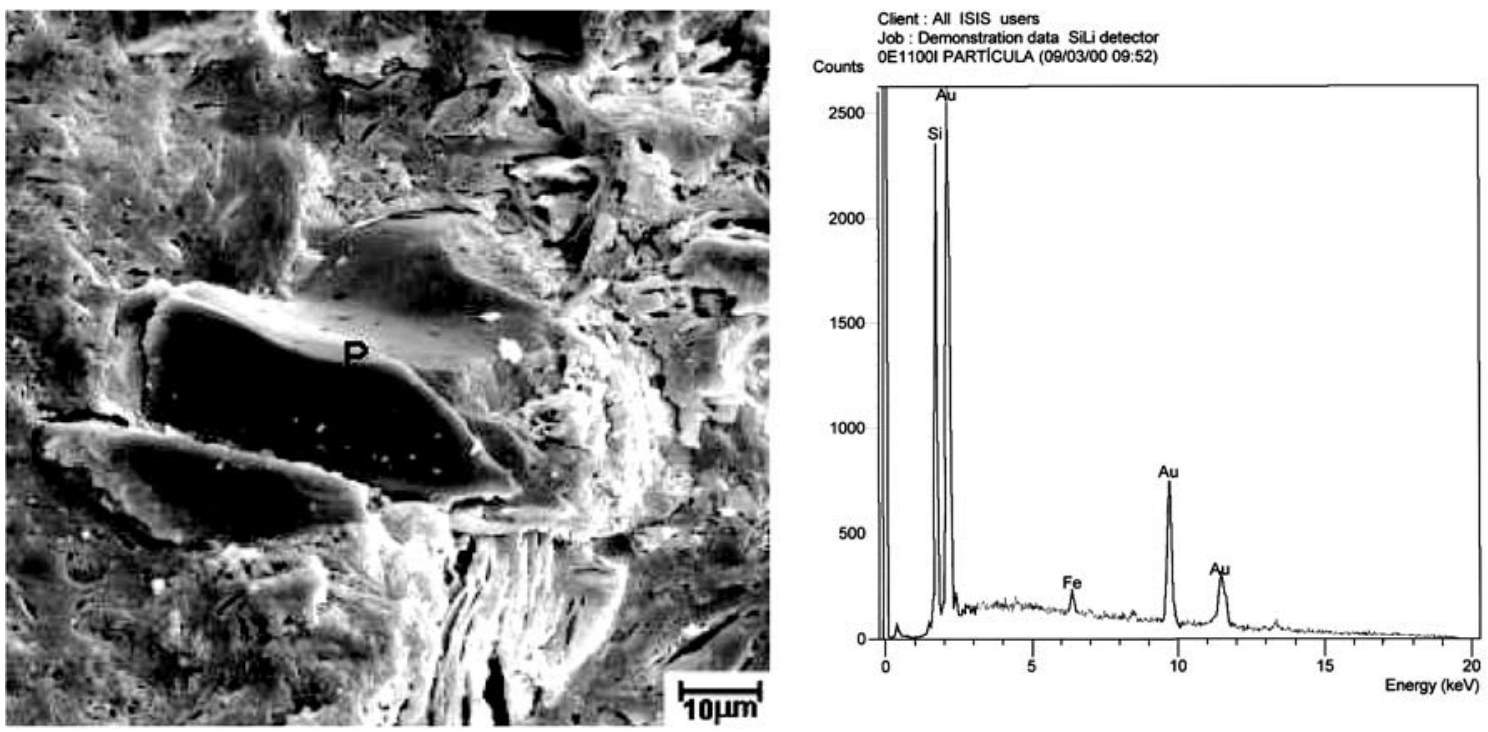

Figura 4: Micrografia obtida por MEV da cerâmica sem resíduo oleoso inertizado queimada a $1100{ }^{\circ} \mathrm{C}$ com EDS mostrando partícula $\mathrm{P}$ composta predominantemente de Si.

[Figure 4: SEM micrograph of the waste-free clay fired at $1100^{\circ} \mathrm{C}$ with EDS showing a particle P predominantly composed of Si.] 

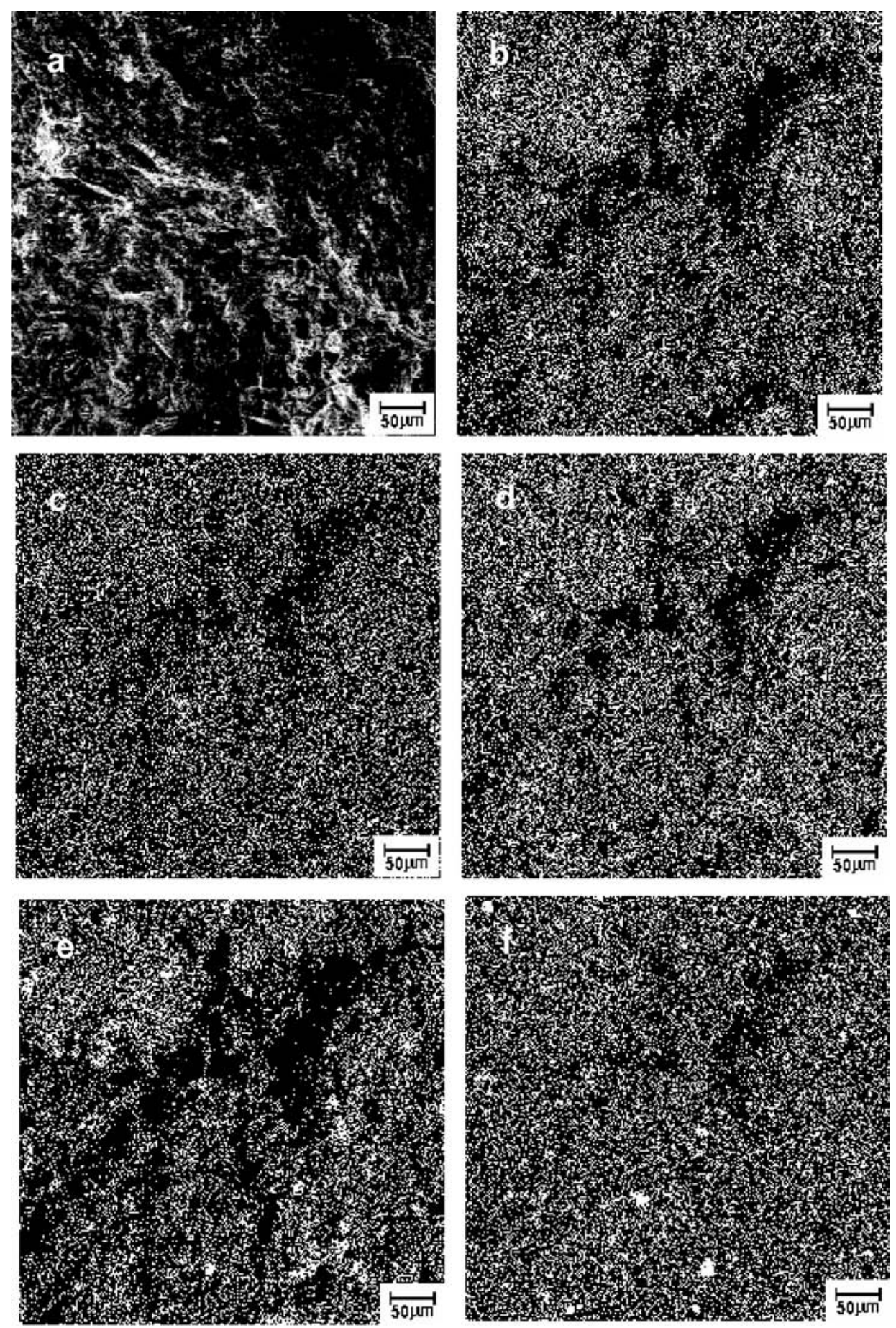

Figura 5: Micrografias obtidas por MEV da cerâmica sem resíduo oleoso inertizado queimada a $1100{ }^{\circ} \mathrm{C}$. (a) microestrutura da cerâmica; (b) mapeamento de $\mathrm{Al}$; (c) mapeamento de $\mathrm{Ca}$; (d) mapeamento de $\mathrm{K}$; (e) mapeamento de $\mathrm{Si}$; (f) mapeamento de Ti.

[Figure 5: SEM micrograph of the waste-free clay without inert oily waste fired at $1100^{\circ} \mathrm{C}$. a) microstructure of the ceramic; (b) Al mapping; (c) Ca mapping; (d) K mapping; (e) Si mapping; (f) Ti mapping.]

de borra encapsulada, pode-se afirmar que elas estão somente associadas aos resíduos oleosos da separação do petróleo. Para justificar a presença de sulfato de bário, vale aqui mencionar que a água pode funcionar como um dos mecanismos naturais para sua produção. Teoricamente, a origem do sulfato de bário pode então ser explicada pelo fato de que, dentre os sais presentes, o sulfato de bário apresenta um dos mais baixos produtos de solubilidade. Sabe-se inclusive, que este sal é responsável por incrustações na coluna de produção e obstruções nos poros das formações produtoras. 

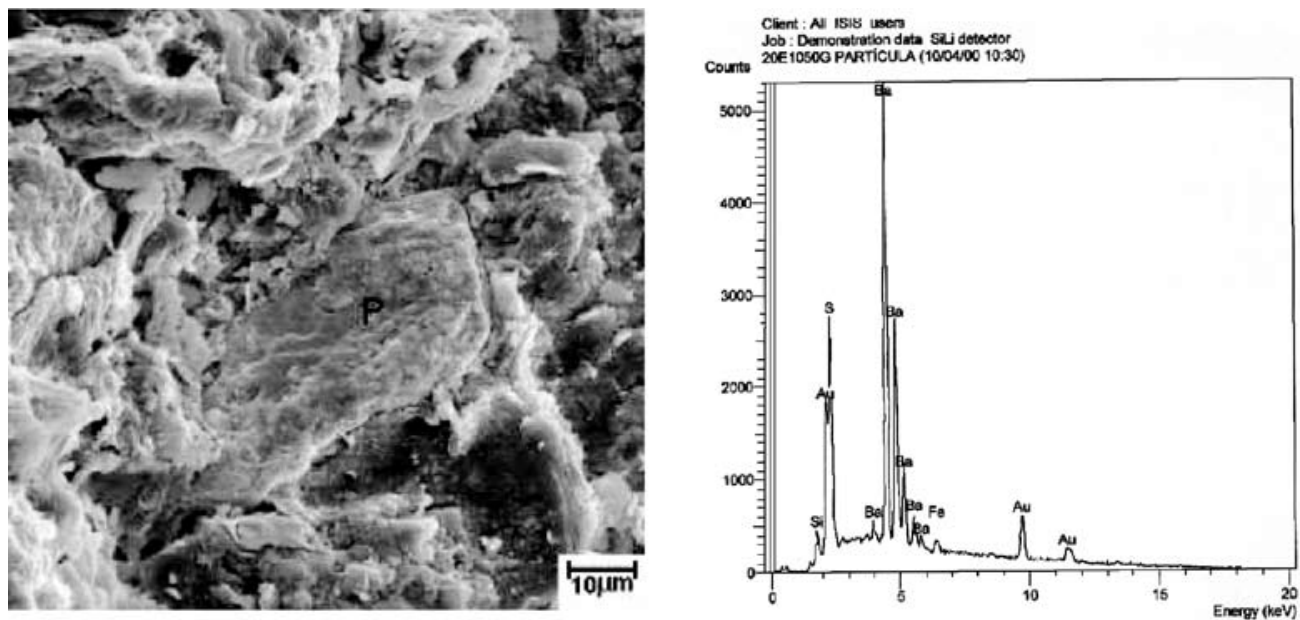

Figura 6: Micrografia obtida por MEV da cerâmica com incorporação de $20 \%$ em peso de resíduo oleoso inertizado queimada a $1050{ }^{\circ} \mathrm{C}$ com EDS mostrando partícula $\mathbf{P}$ composta predominantemente de Ba.

[Figure 6: SEM micrograph of the clay with incorporation of $20 \mathrm{wt} \%$ of oily waste fired at $1050^{\circ} \mathrm{C}$ with EDS showing a particle $\boldsymbol{P}$ predominantly composed of Ba.]
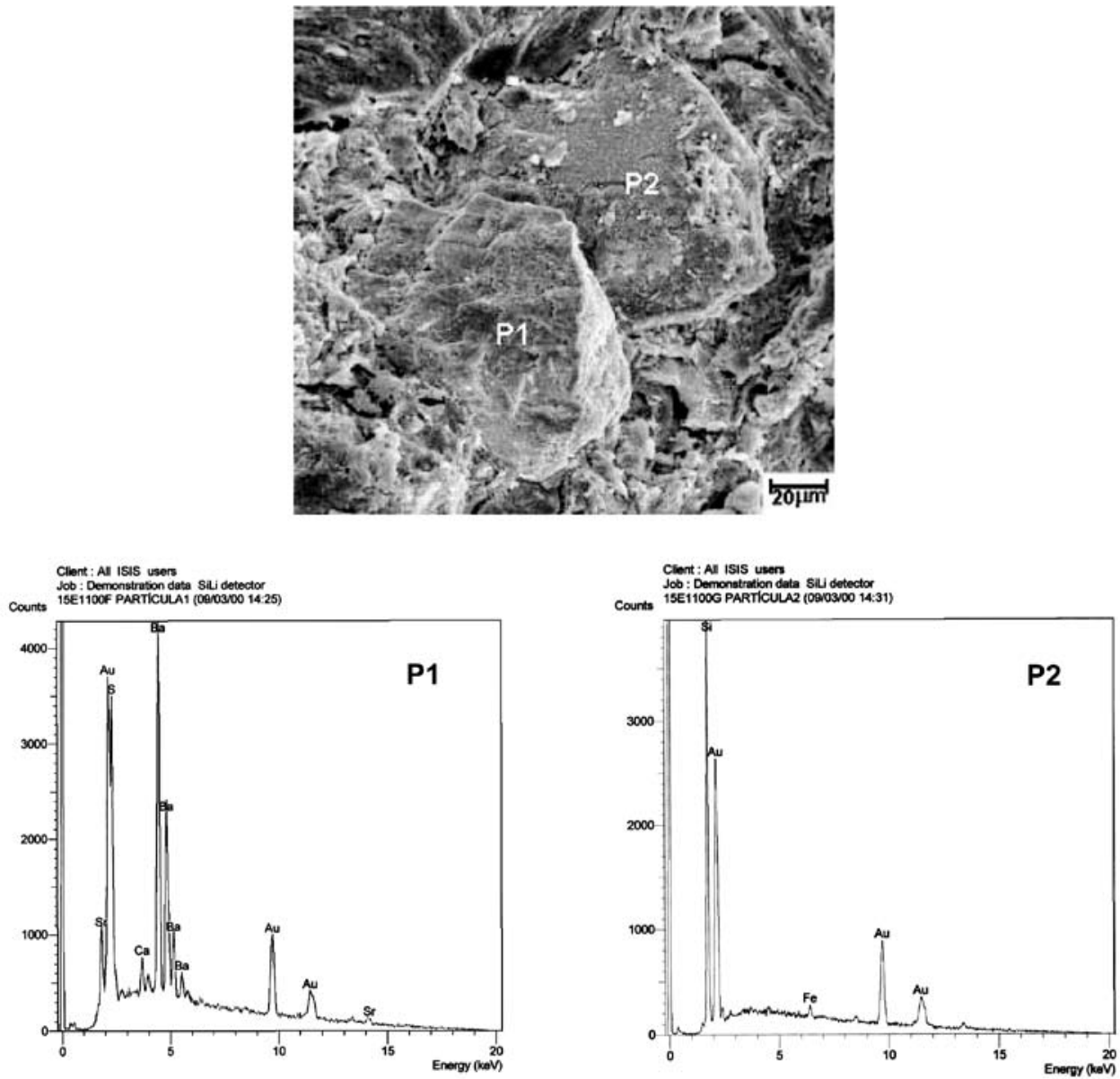

Figura 7: Micrografia obtida por MEV da cerâmica com incorporação de $15 \%$ em peso de resíduo oleoso inertizado queimada a $1100{ }^{\circ} \mathrm{C}$ com EDS mostrando partícula $\mathbf{P 1}$ composta predominantemente de Ba e partícula $\mathbf{P} 2$ de Si.

[Figure 7: SEM micrograph of the clay with incorporation of 15 wt.\% of oily waste fired at $1100{ }^{\circ} \mathrm{C}$ with EDS showing a particle P1 predominantly composed of Ba and $\mathbf{P} 2$ composed of Si.] 

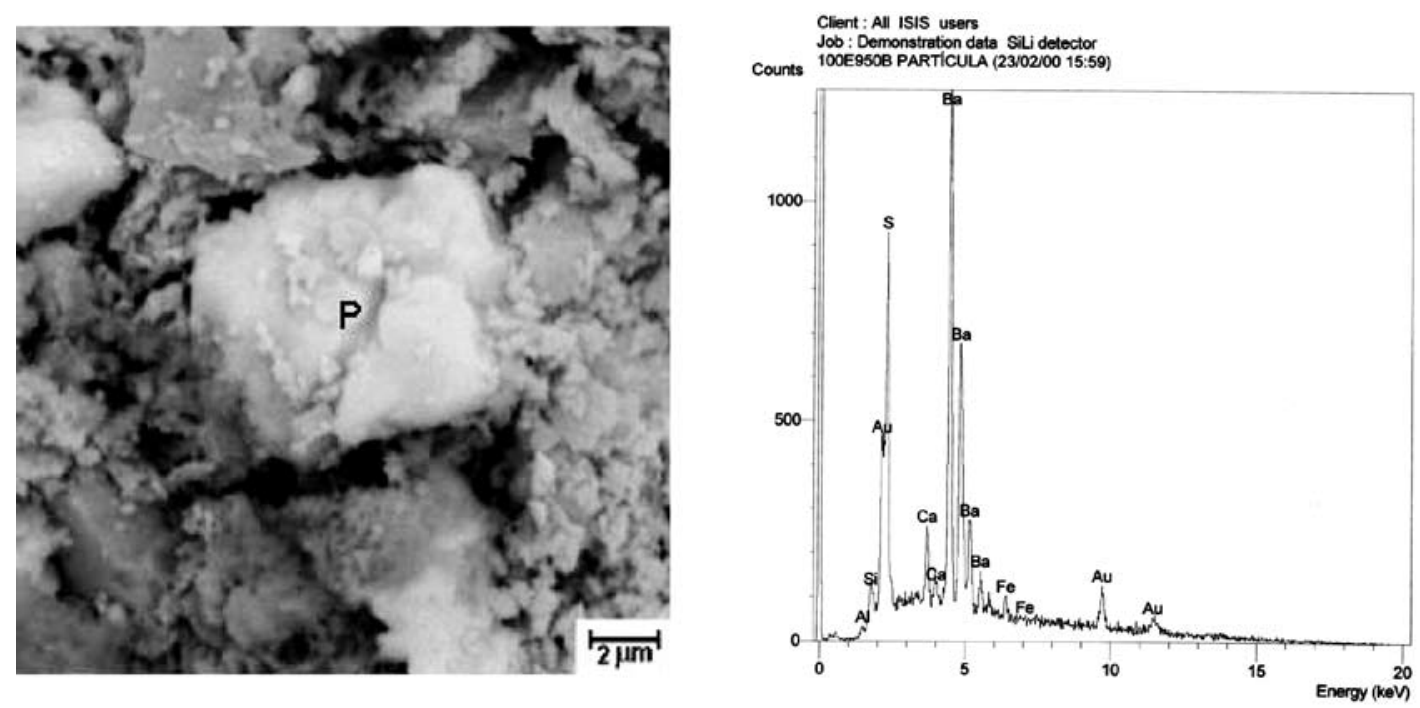

Figura 8: Micrografia obtida por MEV do resíduo oleoso inertizado queimado a $950{ }^{\circ} \mathrm{C}$ com EDS mostrando partícula $\mathrm{P}$ composta predominantemente de Ba.

[Figure 8: SEM micrograph of the inert oily waste fired at $950^{\circ} \mathrm{C}$ with EDS showing a particle $\boldsymbol{P}$ predominantly composed of Ba.]

Partículas de sulfato de bário foram observadas em todas as superfícies de ruptura dos corpos de prova que continham algum percentual de resíduo, participando da fratura do material. É importante ressaltar que as partículas ricas em bário, como na Fig. 5, apresentam formato poliedral com arestas que podem servir como concentradores de tensão para nucleação de trincas [5]. Ou seja, as partículas de sulfato de bário poderiam ser, pelo menos parcialmente, responsáveis pelo decréscimo da resistência mecânica da cerâmica quanto maior for a quantidade incorporada de borra de petróleo encapsulada.

\section{CONCLUSÕES}

A análise microestrutural da superfície de fratura dos corpos de prova mostra que, após a adição de borra de petróleo encapsulada, partículas de composição, formato e características distintas das observadas no material sem adição do resíduo, passam a participar do processo de ruptura do material. Aparentemente, a maior quantidade de partículas observadas poderia aumentar os pontos de nucleação de trincas e acarreta perda de resistência do material para maiores incorporações de borra de petróleo encapsulada.

As análises por EDS demonstram que as principais partículas observadas no material cerâmico, após a incorporação do resíduo de borra de petróleo encapsulada, são sulfato de bário. Este sulfato de bário está associado à água que sai misturada com o petróleo no processo de extração. Partículas de óxido de silício, óxido de ferro, óxido de titânio, alumínio, ferro, cálcio e potássio, são encontradas também no material sem incorporação do resíduo.

\section{AGRADECIMENTOS}

Os autores agradecem o apoio a esta pesquisa concedido pela FINEP através do projeto 0573/96. É também motivo de agradecimento a colaboração de Rogério S. Santos e do Prof. Eduardo A. Carvalho. O suporte da FENORTE, CAPES e CNPq também deve ser reconhecido.

\section{REFERÊNCIAS}

[1] S. P. Amaral, G. H. Domingues, "Aplicação de resíduos Oleosos na Fabricação de Materiais Cerâmicos", Anais do $4^{\circ}$ Cong. Bras. Petróleo, IBP, Rio de Janeiro (1990) 13.

[2] S. N. Monteiro, E. A. Carvallho, E. M. S. Oliveira, N. L. Mendes, "Use of oily waste in structural ceramics - an environmental solution”, in: Proc. Global Symp. Recycling. Waste Treatment and Clean Techn., San Sebastian, 1 (1999) 745.

[3] S. N. Monteiro, E. M. S. Oliveira, E. A. Carvalho, R. S. Santos, "Structural changes related to the addition of oily waste in red clay ceramics", Acta Microscopica 8 (1999) 73.

[4] J. N. F. Holanda, G. P. Souza, "Caracterização de argilas da baixada Campista - RJ por difração de raios X", Anais do $43^{\circ}$ Cong. Bras. Cerâm., Florianópolis, SC (1999) cdrom.

[5] G. P. Souza, R. S. Santos, J. N. F. Holanda, "Recycling of a petroleum waste in ceramic bodies”, Mater. Sci. Forum 418 (2003) 743.

[6] G. P. Souza, J. N. F. Holanda, "Densification behaviour of petroleum waste bearing clay-based ceramic bodies", Ceram. Int. 30, 1 (2004) 99.

[7] S. N. Monteiro, C. M. F. Vieira, "Effect of oily waste addition to clay ceramic”, Ceram. Int. 31, 2 (2005) 353.

[8] Petróleo Brasileiro S/A - Petrobrás, "Relatório Tratamento de borra oleosa”, Rio de Janeiro (1998). (Rec. 03/02/2005, Ac. 06/05/2005) 